\title{
Quantitative approximation by nonlinear convolution operators of Landau-Choquet type
}

\author{
SORIN G. GAL and IONUŢ T. IANCU
}

\begin{abstract}
.
By using the concept of Choquet nonlinear integral with respect to a monotone set function, we introduce the nonlinear convolution operators of Landau-Choquet type, with respect to a family of submodular set functions. Quantitative approximation results in terms of the modulus of continuity are obtained with respect to some particular possibility measures. For some subclasses of functions we prove that these Landau-Choquet type operators can have essentially better approximation properties than their classical correspondents.
\end{abstract}

Acknowledgement. We thank the referees for their useful comments.

\section{REFERENCES}

[1] Choquet, G., Theory of capacities, Ann. Inst. Fourier (Grenoble), 5 (1953-1954), 131-292

[2] Denneberg, D., Non-Additive Measure and Integral, Kluwer Academic Publisher, Dordrecht, Boston, London, 2010

[3] Dubois, D. and Prade, H., Possibility Theory, Plenum Press, New York, 1988

[4] Feller, W., An Introduction to Probability Theory and Its Applications II, Wiley, New York, 1966

[5] Gal, S. G., Approximation by nonlinear Choquet integral operators, Annali di Mat. Pura Appl., 195 (2016), No. 3, 881-896

[6] Gal, S, G., Uniform and pointwise quantitative approximation by Kantorovich-Choquet type integral operators with respect to monotone and submodular set functions, Mediterr. J. Math., 14 (2017), No. 5, 205-216

[7] Gal, S. G., Quantitative approximation by nonlinear Picard-Choquet, Gauss-Weierstrass-Choquet and PoissonCauchy-Choquet singular integrals, Results Math., 73 (2018), No. 3, Art. 92, 23 pp.

[8] Gal, S. G., Shape preserving properties and monotonicity properties of the sequences of Choquet type integral operators, J. Numer. Anal. Approx. Theory, 47 (2018), No. 2, 135-149

[9] Gal, S. G., Quantitative approximation by Stancu-Durrmeyer-Choquet-Sipos operators, Math. Slovaca, 69 (2019), No. 3, 625-638

[10] Gal, S. G. and Opris, B. D., Uniform and pointwise convergence of Bernstein-Durrmeyer operators with respect to monotone and submodular set functions, J. Math. Anal. Appl., 424 (2015), 1374-1379

[11] Gal, S. G. and Trifa, S., Quantitative estimates in uniform and pointwise approximation by Bernstein-Durrmeyer-Choquet operators, Carpath. J. Math., 33 (2017), 49-58

[12] Gal, S. G. and Trifa, S., Quantitative estimates in $L^{p}$-approximation by Bernstein-Durrmeyer-Choquet operators with respect to distorted Borel measures, Results Math., 72 (2017), No. 3, 1405-1415

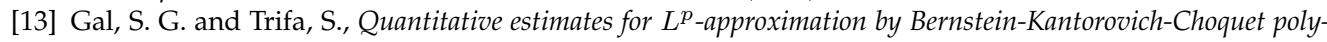
nomials with respect to distorted Lebesgue measures, Constructive Mathematical Analysis, 2 (2019), No. 1, $15-21$

[14] Jackson, D., A Proof of Weierstrass's Theorem, Amer. Math. Monthly, 41 (1934), No. 5, 309-312

[15] Landau, E., Über die Approximation einer stetigen Funktion durche eine ganze rationale Funktion, Rendicontdi del Circolo Matematico di Palermo, 25 (1908), 337-345

Received: 26.10.2019; In revised form: 30.01.2020; Accepted: 06.02.2020

2010 Mathematics Subject Classification. 41A36, 41A25, 28A10, 28A12, 28A25.

Key words and phrases. monotone set function, possibility measure, nonlinear Choquet integral, nonlinear Landau-Choquet operators, modulus of continuity.

Corresponding author: Sorin G. Gal; galso@uoradea.ro 
[16] Sugeno, M., Theory of Fuzzy Integrals and its Applications, Ph.D. dissertation. Tokyo Institute of Technology, Tokyo, 1974

[17] Wang, S. and Klir, G. J., Generalized Measure Theory, Springer, New York, 2009

[18] Weierstrass, K., Über die analytische Darstellbarkeit sogenannter willkürlicher Functionen einer reellen Veränderlichen, Sitzungsberichte der Akademie zu Berlin, (1885), pp. 633-639 and 789-805

Department of Mathematics And COMPuter SCiEnCE

UNIVERSITY OF ORADEA

UNIVERSITĂŢII 1, 410087 ORADEA, ROMANIA

Email address: galso@uoradea.ro

Email address: ionutz.tudor. ianculgmail.com 